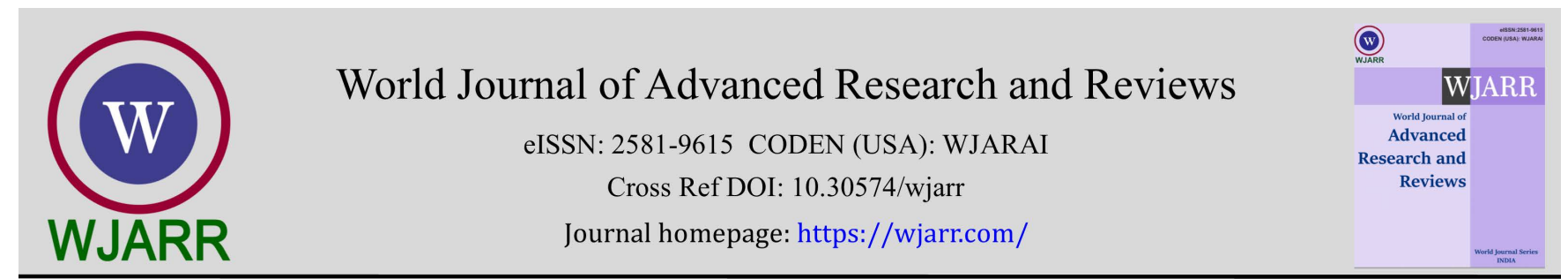

(REVIEW ARTICLE)

\title{
Pacemaker associated reduction of left ventricle systolic function
}

\author{
Ivaneta D Yoncheva ${ }^{1,}{ }^{*}$, Denislav E Biserov ${ }^{1}$ and Mariya N Negreva ${ }^{2}$ \\ ${ }^{1}$ University Hospital Virgin Mary, Burgas, Bulgaria. \\ ${ }^{2}$ University Hospital St. Marina, Varna, Bulgaria.
}

World Journal of Advanced Research and Reviews, 2021, 12(02), 048-054

Publication history: Received on 27 September 2021; revised on 31 October 2021; accepted on 02 November 2021

Article DOI: https://doi.org/10.30574/wjarr.2021.12.2.0563

\begin{abstract}
In recent years, data have been accumulated on the negative effect of right ventricular (RV) stimulation, leading to left ventricular (LV) asynchrony, proarrhythmias and progressive heart failure (HF). On the other hand, biventricular pacing has been shown to affect ventricular asynchrony, reduce HF manifestations, and improve prognosis in patients with LV dysfunction and wide QRS complex.

The induced asynchrony from apical right ventricular pacing is unequivocally associated with changes in myocardial perfusion, LV dysfunction, and poorer prognosis for patients over time. This has led researchers for decades to look for an alternative position for electrode placement.

The incidence of pacemaker-induced cardiomyopathy (PICM) ranges from 5.9 to 39\% in patients with RV pacing, depending on the given definition and the limit for the degree of pacing. Upgrading to biventricular pacing has been shown to reverse the cardiomyopathy. Recently, there has been evidence of a positive effect of His bundle pacing (HBP) in the treatment of PICM even in patients with no improvement after biventricular pacing.

The question about the pathogenetic mechanisms of PICM is currently unanswered. The connection between electrical asynchrony and the negative effect on cardiac pump function is clear. There is also evidence of an established relationship between asynchrony and coronary blood flow. The predisposing individual characteristics of the patient in which these negative effects are manifested are not clear. This is an issue that requires further studies.
\end{abstract}

Keywords: Asynchrony; Cardiostimulation; Cardiomyopathy; QRS duration

\section{Introduction}

According to pacemaker manufacturers, 355,000 devices are implanted annually in Europe, corresponding to 878 implantations per 1 million people [1]. Indication for primary implantation in about half of the patients is permanent or intermittent atrioventricular block, leading respectively to apical RV pacing with a varying percentage [2]. In about $50 \%$ of cases, ventricular pacing can be minimized with different device algorithms [3]. This means that 88,000 patients in Europe each year will have permanent RV pacing, which can lead to adverse effects on cardiac pump function.

In recent years, data have been accumulated on the negative effect of RV pacing, leading to LV asynchrony [4], proarrhythmias and progressive HF [5].

On the other hand, biventricular pacing has been shown to affect ventricular asynchrony, reduce HF manifestations, and improve prognosis in patients with LV dysfunction and wide QRS complex [6].

\footnotetext{
${ }^{*}$ Corresponding author: Ivaneta D Yoncheva

University Hospital Virgin Mary, Burgas , Bulgaria.

Copyright (C) 2021 Author(s) retain the copyright of this article. This article is published under the terms of the Creative Commons Attribution Liscense 4.0.
} 
Data from resynchronization therapy studies confirm the hypothesis of the positive effect of biventricular stimulation on LV pump function compared to that in RV apex in high-grade AV block patients.

The PREVENT-HF study compared randomized patients with dual chamber or biventricular pacing with normal LVEF at baseline and high-grade AV block [1]. They were monitored by echocardiography at 6 and 12 months, with no significant difference in LV volume and ejection fraction (EF). However, in order to obtain more convincing data, it is necessary to cover a larger number of patients, as well as to monitor the patients for a longer period of time.

The optimal pacing mode in sick sinus syndrome (SSS) is still debatable. Achieving atrioventricular synchrony with dual chamber pacemakers has an advantage by default, which, however, has turned out difficult to prove by years of research on this patient population.

Using data from large randomized trials, a consensus was reached on the lack of survival advantage in patients with dual compared to single chamber pacemakers. At the same time, data from the same studies provide conflicting results on the effect of the pacing mode on cardiovascular morbidity. A possible explanation might be that ventricular asynchrony caused by constant apical RV pacing neutralizes the positive effects of the achieved atrioventricular synchrony.

In 2003 Sweeney et al. analyzed 2010 patients included in the MOde Selection Trial (MOST), a six-year randomized prospective study comparing patients with SSS and implanted single or dual chamber pacemakers [7]. This study tested the theory that ventricular asynchrony induced by apical RV pacing, even with AV synchrony present, resulted in increased hospitalizations for decompensated HF and atrial fibrillation (AF) in patients with SSS and normal baseline QRS complex width. Cumulative percent ventricular paced (Cum\%VP) was determined from stored pacemaker data. Baseline QRS duration data were available in in 1732 of 2010 patients (86.2\%). Baseline QRS duration <120 ms was observed in 1339 of 1732 (77.3\%). Of these 1339, 707 (52.8\%) were randomly assigned to DDDR and 632 (47.2\%) to VVIR. Most patients had normal left ventricular function (median ejection fraction, 55\%) with no symptoms of congestive heart failure. More than half had a history of atrial tachycardia. The baseline PR interval was normal $(<200$ $\mathrm{ms}$ ) or mildly prolonged in most patients. Dividing the patients into groups according to Cum $\% \mathrm{VP}$ above $40 \%$ and below $40 \%$, obtained HR [CI] of 2.60 [1.05-6.47] ( $\mathrm{P}=0.040)$. This shows that ventricular pacing over $40 \%$ of the time in the DDDR mode was associated with a 2.6-fold increased risk of hospitalization for HF compared with pacing $<40 \%$.

Analysis of the data showed an increase in the risk of AF with the increase in Cum\%VP, both in the DDDR and VVIR group. The magnitude of increased risk was $1 \%$ for each $1 \%$ increase in Cum $\%$ VP and reached up to $85 \%$ in DDDR mode.

Data from this study indicate a significant advantage in SSS patients with atrial pacing compared to the DDDR mode, as a result of avoidance of asynchronous ventricular activation resembling left bundle-branch block.

The effect of permanent pacing on LV pump function as well as arrhythmias are of interest to researchers and are the topic of many studies in the field. To develop new therapeutic possibilities and to understand the pathogenetic mechanisms of HF, it is necessary to conduct animal studies. The pig is a suitable research animal, given the similarities in cardiovascular anatomy, physiology and size compared to humans. Arterial pressures in the systemic and pulmonary circulation system as well as a stable sinus rhythm match those of human conditions [8]. Various techniques are used to induce heart failure in large experimental animals, with rapid ventricular pacing being the most preferred method [9]. This method can induce LV dilatation and remodeling, changes in hemodynamic parameters and clinical manifestations of HF.

A study performed on pigs by Möllmann et al. aimed to prove the association between ventricular asynchronous contraction and development of HF. They used two groups of 10 male pigs and 6 sham-operated served as controls. The animals in group A

received a specially programmed VVI pacemaker, those in group B received a dual chamber DDD pacemaker with both leads implanted in different RV regions, causing complete desynchronization of the ventricular contraction. All pigs were paced for 3 weeks at $220 \mathrm{bpm}$ followed by a 1-week recovery period after termination of pacing. Before implantation and after the period of rapid ventricular pacing, chocardiography was performed and hemodynamic parameters were evaluated. Also at the end of the follow-up period, the animals were euthanized and ventricular myocardial samples were taken for immunohistochemical analysis. 
There was a significantly lower CI (cardiac index) in group B than in group A $(2.9 \pm 0.2 \mathrm{l} / \mathrm{min} / \mathrm{m} 2 \mathrm{vs} .3 .6 \pm 0.2 \mathrm{l} / \mathrm{min} / \mathrm{m} 2$, $\mathrm{p}<0.05$; controls $4.3 \pm 0.1 \mathrm{l} / \mathrm{min} / \mathrm{m} 2$ ). The reduction in CI was not associated with decreased mean pulmonary artery pressure in either group. In contrast, however, there was a significant increase in central venous pressure and pulmonary capillary pressure after rapid ventricular pacing with significantly higher values in group B compared to A. Examination of the heart muscle showed a tendency to spherical LV remodeling, as well as dilatation of the four cardiac chambers after rapid ventricular pacing. Analysis of collagen content in the hearts with induced pump failure found increased interstitial fibrosis and thickened collagen fibrils, which in turn lead to myocyte disintegration. Rapid ventricular pacing lead to an increased LV diameter, as well as increase in the amounts of collagen 3 and 6 in the group with pronounced asynchrony with two pacing electrodes. This shows that fibrosis is an important determinant for the development of HF, an issue discussed in previous publications [10].

The induced asynchrony from apical RV pacing is unequivocally associated with changes in myocardial perfusion, LV dysfunction, and poorer prognosis for patients over time [11]. This has led researchers for decades to look for an alternative position for electrode placement. A large meta-analysis of 985 randomized clinical trials performed by Shimony et al. aimed to compare the advantages of RVNAP (right ventricular non-apical pacing sites) and RVAP (right ventricular apical pacing) [12]. The change in LVEF was assessed in 13 of the studies, with the analysis showing significantly higher LVEF in patients with RVNAP compared to those with RVAP. It is important to note that baseline systolic function did not differ significantly in both groups of patients. One study compared long-term survival (120 months) in RVNAP and RVAP and found no significant difference in this indicator [13]. The meta-analysis showed that the degree of benefit in RVNAP remains persistent over time. As an end result, the limitation of the short follow-up of patients in most studies remains. However, the conclusion is clear for worsened prognosis in patients with RVAP and baseline reduced $\mathrm{LVEF}<45 \%$ [14].

Permanent RV apical pacing is one of the determinants of change in LV pump function over time. However, it is not clear for how long these negative effects manifest themselves. In addition to asynchronous ventricular contraction, AV synchronization and consequent AF manifestation are also important. A number of studies were performed to identify the optimal mode of programming the pacemaker and its effect on AF manifestation or LV dysfunction. However, data from these studies are quite contradictory.

The pathophysiology of AF is complex, and its manifestation requires a triggering factor and substrate in the atrial myocardium [15]As end-diastolic pressure (EDP) increases in LV, left atrial pressure increases to ensure adequate diastolic filling. This leads to increased stress of the left atrial myocardium, structural remodeling, dilatation and fibrosis, which in turn become a substrate for the manifestation of AF.

Data from studies in patients with implanted CRT show that overcoming LV ventricular asynchrony leads to improved LV pump parameters. This is associated with reverse remodeling of both left ventricular and left atrial myocardium, which has been associated with a reduction in AF incidents in these patients as well as stabilization of heart rhythm [16].

The association between AF and RV apical pacing was also demonstrated in the SAVE PACe study [17]. 1065 SSS patients were included and the effect of dual-chamber pacemakers with a function to minimize RV pacing compared to conventional dual-chamber devices. The primary endpoint was to register persistent AF for a follow-up period of 1.7 years. Persistent AF was detected in $12.7 \%$ of patients with a conventional dual-chamber pacemaker and in $7.9 \%$ of the group with a RV pacing minimization algorithm $(\mathrm{P}=0.004)$. Mortality and hospitalizations for $\mathrm{HF}$ were comparable in both groups.

Asynchronous ventricular contraction leads to decreased stroke volume, functional mitral regurgitation, and inefficient LV emptying. Besides apical RV pacing, asynchrony is observed in the presence of a block in the conduction system, as well as in ventricular ectopia. To establish a link between impaired ventricular systolic function and ectopic site contraction, Walters et al. at the University of California, San Francisco, conducted a study with pigs. [18] The study included 20 experimental animals and had two phases. In the first phase, one group was exposed to paced bigeminy from the right ventricular apex for 14 weeks $(n=10)$ and was compared with a group exposed to regular pacing from the RVA at 140 beats/min $(n=5)$ and a control group $(n=5)$. The second phase compared the effect of ventricular ectopia from different points on the change in LV function over 12 weeks. The experimental animals were divided into 4 groups of 5: paced ventricular extrasystoles in bigeminy from the free RV wall, lateral LV wall, right atrial ear, and control group.

Hemodynamic parameters were measured invasively at baseline and at the end of the study protocol. Echocardiography follow-up revealed a significant reduction in LVEF in the group with RV apical ectopia (from 67.8 to 45.3\%; $\mathrm{p}<0.05$ ), 
but no significant difference in the control group. In phase 2, there was a significant reduction in LVEF in both groups with ectopia from the free wall of the RV and LV epicardium, and this decrease was more pronounced in the second group. After completion of the follow-up protocol, experimental animals were euthanized and histological samples were taken from various parts of the heart muscle. The results showed an increase in myocardial fibrosis in those with ventricular ectopia, with the most pronounced change observed again in the group with extrasystoles from the epicardium of the LV. Published epidemiological studies clearly define the relationship between ventricular ectopia and the risk of cardiomyopathy and HF [19], as well as the fact that their effective suppression leads to reversal of the disorders [20].

The belief that LV asynchrony may have adverse effects on long-term prognosis in paced patients has led researchers to experiment with alternative points of stimulation.

Animal studies have shown that pacing the LV septum maintains pump function close to normal and is significantly better compared to RV septal pacing [21]. In animal models, the electrode is placed intravenously in the RV, from where the LV septal surface is reached transseptally with a longer than the standard fixing helix. Monitoring shows that the electrodes remain mechanically and electrically stable for the observed period in otherwise healthy and active dogs.

Based on these data, Mafi-Rad et al. conducted a study in humans, examining applicability of transvenous access for electrode placement on the LV septum, as well as comparing the effect on hemodynamics in LV septal pacing, RV septal pacing and conventional RVA pacing. [22]. They included patients with indications for permanent pacemaker implantation in SSS who were not expected to be pacemaker-dependent, suggesting a low risk of complication of pacing electrode dysfunction. For this purpose, a modified electrode with a longer fixing helix of $4 \mathrm{~mm}$ was used to ensure good penetration and stability in the interventricular septum. Also, the fixing part of the electrode was partially covered with insulation, leaving uncovered only the top part of $1.27 \mathrm{~mm}$ to perform the pacing. The correct position of the electrode was confirmed by fluoroscopy and intracardiac echocardiography. After its placement, the pacing threshold of electrode impedance was measured, and changes in LV dP/dt in comparison with the AAI mode were determined. Patients were monitored by measuring electrode sensing, pacing threshold, and impedance immediately after implantation, at 10 days, 1 month, 3 months, and 6 months. There were no significant changes in these parameters for the follow-up period. Right ventricular apical (RVA) and right ventricular septal pacing (RVS) reduce LV dP/dt max compared to atrial pacing, while LVS pacing does not result in such a change. These changes were present in all studied patients. In this study, a shortening of the electrical asynchrony was observed, which led to a shortening of the QRS complex compared to RVA and RVS. During normal electrical myocardial activation, ventricular excitation began from the LV portion of the septum, which might explain why pacing near that portion mimics normal activation as well as normal mechanical synchronicity.

A significant finding of this study is the large difference in the duration of the QRS complex and hemodynamic effects in interventricular RV and LV septal pacing. Although the difference between the two pacing points was less than $10 \mathrm{~mm}$, pacing from RV lead to a delay in transseptal impulse propagation, which slowed the excitation of the free LV wall and resulted in intraventricular and interventricular asynchrony.

Another alternative pacing is the His bundle (HB). This method was first described by Deshmukh in a small population of AF and dilated cardiomyopathy (DCM) patients in 2000 [23]. In recent years, the benefits and safety of this method have been unequivocally proven in many studies as a physiological alternative to RV apical pacing.

For the period 2013-2016, Abdelrahman et al. compared two groups of patients with standard indications for permanent pacemaker implantation [24]. HB was paced in 332 of them, in 433 the RV apex. The implantations were performed in two separate hospitals in Pennsylvania. Patients were followed-up every 2 weeks, every 2 months and annually. The primary endpoint was death for any reason, hospitalization for HF, and need for an upgrade to a biventricular pacemaker. The mean baseline EF was $54.5 \pm 9.5 \%$ and the mean QRS duration was $108 \pm 27$ ms. During the follow-up period, there were 117 hospitalizations due to decompensated HF, with 104 hospital stays over 24 hours, and in 96 patients $>48$ hours. Significantly fewer hospitalizations were observed in patients with HB pacing (41 of 332 [12.4\%]) compared to RVP (76 of 433 [17.6\%]; HR: 0.633; $\mathrm{p}=0.02$ ). This difference was observed in patients with ventricular pacing $>20 \%$. The results of this study show that HB pacing was associated with a significant reduction in overall mortality, need for HF hospitalization, and need for reimplantation with a biventricular device. They also proved that HB pacing can prevent ventricular asynchrony due to propagation of the stimulated impulse along the native HisPurkinje conduction system.

A recently published metanalysis by Fernandes et al. in 2020 provides comprehensive information on the effect of different RV stimulation points on LV pump function, patients' physical capacity, hospitalizations for decompensated $\mathrm{HF}$, and overall mortality [25]. 10 studies were included - in 6 of them BiVP and RVP were compared, and in 4 HBP and 
RVP. BiVP and HBP were associated with a significantly lower risk of overall mortality and HF hospitalization compared to RVP [OR 0.66]. In studies evaluating the change in the LVEF, there is a clear advantage of BiVP and HBP over RVP, both in impaired AV conduction and in those after His ablation.

It is already well known that pacing the RV apex leads to LVEF reduction. This results in PICM, usually defined as FI below 40\%, excluding other causes. The incidence of PICM varies from 5.9 to $39 \%$ in patients with RV pacing depending on the given definition and limit for pacing degree [26]. Upgrading to biventricular stimulation has been shown to reverse the cardiomyopathy. Recently, there is evidence of a positive effect of HB pacing in the treatment of PICM even in patients with no improvement after biventricular pacing [27].

This metanalysis demonstrates that the primary strategy of HBP and BiVP in patients with normal or borderline EF reduces the incidence of hospitalizations for decompensated HF by 39\%. Since the nosological status of PICM is relatively unclear, it is not established whether adverse effects occur only in patients who have developed cardiomyopathy or whether there are other subclinical causes that are provoked by RV pacing.

Study data suggest a significant correlation between the duration of the paced QRS complex and long-term prognosis. According to Kim et al., QRS duration above $140 \mathrm{msec}$ has a 95\% sensitivity, and that above $167 \%$ has a $90 \%$ specificity for PICM development for an average follow-up period of 4.5 years in South Korea [28].

Similar results were obtained by Khurshid et al., the duration of QRS over $150 \mathrm{msec}$ being a highly sensitive marker for the development of PICM. Also, these patients should be monitored echocardiographically to register a decrease in LVEF before symptoms of decompensated HF appear [29].

Data from previous studies indicate that there is impaired regional perfusion of the interventricular septum in patients with left bundle branch block (LBBD) [30]. There is also evidence of improved blood flow in LAD after implantation of a resynchronization therapy system in patients with DCM, while permanent RV pacing leads to its reduction [31].

\section{Conclusion}

The question of the pathogenetic mechanisms of PICM is currently unanswered. The relationship between electrical asynchrony and negative effect on the pump function of the heart is evident. There is also evidence of a relationship between asynchrony and coronary blood flow. The predisposing individual characteristics of the patients in which these adverse effects are manifested, as well as the profibrotic changes in the intercellular matrix in the affected individuals, are not clear. To answer these questions, it is necessary to conduct in-depth studies at cellular and subcellular level in the heart muscle. We should seek a link between compromised coronary blood flow and cardiomyocyte response and how these interactions are affected by electrical activation of the heart.

The answers to these questions will allow selection of the risk cohort of patients for which to undertake individual pacing strategy and prevention of future complications.

\section{Compliance with ethical standards}

\section{Acknowledgments}

The authors gratefully acknowledge the University Hospital “Virgin Mary” for funding this work.

\section{Disclosure of conflict of interest}

The authors declare that there is no conflict of interests regarding the publication of this paper.

\section{References}

[1] Stockburger M, Gómez-Doblas JJ, Lamas G, Alzueta J, Fernández-Lozano I, Cobo E, et al.Preventing ventricular dysfunction in pacemaker patients without advanced heart failure: results from a multicentre international randomized trial (PREVENT-HF). 2011; 13(6): 633-641.

[2] Markewitz A. Yearly report for 2007 of the German Pacemaker registry. Herzschrittmacherther Elektrophysiology. 2008; 19(4): 195-223. 
[3] Gillis AM, Pürerfellner H, Israel CW, Sunthorn H, Kacet S, Anelli-Monti M, et al. Reducing Unnecessary Right Ventricular Pacing with the Managed Ventricular Pacing Mode in Patients with Sinus Node Disease and AV Block. Pacing and Clinical Electrophysiology. 2006; 29: 697-705.

[4] Liu WH, Chen MC, Chen YL, Guo BF, Pan KL, Yang CH, et al. Right ventricular apical pacing acutely impairs left ventricular function and induces mechanical dyssynchrony in patients with sick sinus syndrome: a real-time three-dimensional echocardiographic study. J Am Soc Echocardiogr. 2008; 21(3): 224229.

[5] Stockburger M, Celebi O, Knaus T, Krebs A, Nitardy A, Habedank D, et al. Right ventricular pacing is associated with impaired overall survival, but not with increased incidence of ventricular tachyarrhythmias in routine cardioverter/defibrllator recipients. Europace. 2009; 11: 924-930.

[6] Cleland JGF, Daubert JC, Erdmann E, Freemantle N, Gras D, Kappenberger L, et al. The effect of cardiac resynchronization on morbidity and mortality in heart failure. CARE-HF study. N Engl J Med. 2005; 352: 15391549.

[7] Sweeney M, Hellkamp A, Ellenbigen K, Greenspon AJ, Freedman RA, Lee KL, et al. Adverse Effect of Ventricular Pacing on Heart Failure and Atrial Fibrillation Among Patients With Normal Baseline QRS Duration in a Clinical Trial of Pacemaker Therapy for Sinus Node Dysfunction. Circulation. 2003; 107: 2932-2937.

[8] Möllmann H, Voss S, Nef HM, Lintz M, Oltenau C, Kostin S, et al. Desynchronization:ijepiA Novel Model to Induce Heart Failure. Thorac Cardiov Surg 2009; Thorac Cardiovasc Surg. 2009; 57(8): 441-448.

[9] Vanoli E, Bacchini S, Panigada S, Pentimalli F, Adamson P. Experimental models of heart failure. Eur Heart J Suppl. 2004; 6: F7-F15.

[10] Siu CW, Wang M, Zhang XH, Lau CP, Tse HF. Analysis of ventricular performance as a function of pacing site and mode. Prog Cardiovasc Dis. 2008; 51(2): 171-182.

[11] Freudenberger RS, Wilson AC, Lawrence-Nelson J, Hare JM, Kostis JB. Permanent pacing is a risk factor for the development of heart failure: Myocardial Infarction Data Acquisition System Study Group (MIDAS 9). Am J Cardiol. 2005; 95(5): 671-674.

[12] Shimony A, Eisenberg MJ, Filion KB, Amit G. Beneficial effects of right ventricular non-apical vs. apical pacing: a systematic review and meta-analysis of randomized-controlled trials. EP Europace. 2012; 14(1): 81-91.

[13] Dabrowska-Kugacka A, Lewicka-Nowak E, Tybura S, Wilczek R, Staniewicz J, Zagozdzon P, et al. Survival analysis in patients with preserved left ventricular function and standard indications for permanent cardiac pacing randomized to right ventricular apical or septal outflow tract pacing. Circ J. 2009; 73(10): 1812-1819.

[14] Leong DP, Mitchell AM, Salna I, Brooks AG, Sharma G, Lim HS et al. Long-term mechanical consequences of permanent right ventricular pacing: effect of pacing site. J Cardiovasc Electrophysiol. 2010; 21(10): 1120-1126.

[15] Melduni R, Cullen M . Role of Left Ventricular Diastolic Dysfunction in Predicting Atrial Fibrillation Recurrence after Successful Electrical Cardioversion. J Atr Fibrillation. 2012; 5(4): 654.

[16] Birnie D, Hudnall H, Lemke B, Aonuma K, Lee K, Gasparini M, et al. Continuous optimization of cardiac resynchronization therapy reduces atrial fibrillation in heart failure patients: Results of the Adaptive Cardiac Resynchronization Therapy Trial. Heart Rhythm. 2017; 14(12): 1820-1825.

[17] Sweeney MO, Bank AJ, Nsah E, Koullick M, Zeng QC, Hettrick D, et al. Minimizing ventricular pacing to reduce atrial fibrillation in sinus-node disease:SAVE PACe Trial. N Engl J Med. 2007; 357: 1000-1008.

[18] Walters TE, Rahmutula D, Szilagyi J, Alhede C, Sievers R, Fang Q, et al. Left Ventricular Dyssynchrony Predicts the Cardiomyopathy Associated With Premature Ventricular Contractions. Journal of the American College of Cardiology. 2018; 72(23A): 2870-2882.

[19] Agarwal SK, Simpson RJ, Rautaharju P, Alonso A, Shahar E, Massing M,et al. Relation of ventricular premature complexes to heart failure :Atherosclerosis Risk In Communities -ARIC Study.Am J Cardiol. 2012; 109(1): 105109.

[20] Deyell MW, Park KM, Han Y, Frankel D, Dixit S, Cooper J, et al. Predictors of recovery of left ventricular dysfunction after ablation of frequent ventricular premature depolarizations. Heart Rhythm. 2012; 9(9): 1465-1472.

[21] Mills RW, Cornelussen RN, Mulligan LJ, Strik M, Rademakers LM, Skadsberg ND, et al. Left ventricular septal and left ventricular apical pacing chronically maintain cardiac contractile coordination, pump function and efficiency. Circ Arrhythm Electrophysiol. 2009; 2(5): 571-579. 
[22] Mafi-Rad M, Luermans J, Blaauw Y, Janssen M, Crijns H, Prinzen F, et al. Feasibility and Acute Hemodynamic Effect of Left Ventricular Septal Pacing by Transvenous Approach Through the Interventricular Septum. Circ Arrhythm Electrophysiol. 2016; 9(3).

[23] Deshmukh P, Casavant D, Romanyshyn M, Anderson K. Permanent direct His bundle pacing: a novel approach to cardiac pacing in patients with normal His-Purkinje activation. Circulation. 2000; 101(8): 869-77.

[24] Abdelrahman M, Subzposh FA, Beer D, Durr B, Naperkowski A, Sun H et al. Clinical Outcomes of His Bundle Pacing Compared to Right Ventricular Pacing. J Am Coll Cardiol. 2018; 71(20): 2319- 2330.

[25] Fernandes G, Knijnik L, Lopez J, Rivera M, Fernandes A, Lambrakos L, et al. Network Meta-analysis of His Bundle, Biventricular or Right Ventricular Pacing as a Primary Strategy for Advanced Atrioventricular Conduction Disease with Normal or Mildly Reduced Ejection Fraction. Journal of cardiovascular Electrophysiology. 2020; 31(6): 1482-1492.

[26] Kaye G, Ng JY , Ahmed S, Valencia D, Harrop D, Arnold CT. The Prevalence of Pacing-Induced Cardiomyopathy (PICM) in Patients With Long Term Right Ventricular Pacing - Is it a Matter Of Definition? Heart Lung Circ. 2019; 28(7): 1027-1033.

[27] Shan P, Su L, Zhou X, Wu S, Xu L, Xiao F, et al. Beneficial effects of upgrading to His bundle pacing in chronically paced patients with left ventricular ejection fraction <50. Heart Rhythm. 2018; 15(3): 405-412.

[28] Kim JH, Kang KW, Chin JY, Kim TS, Park JH, Choi YJ, et al. Major determinant of the occurrence of pacing-induced cardiomyopathy in complete atrioventricular block: a multicentre, retrospective analysis over a 15-year period in South Korea. BMJ Open. 2018; 8: e019048.

[29] Khurshid S, Liang JJ, Owens A, Lin D, Schaller R, Epstein A, et al. Longer Paced QRS Duration is Associated With Increased Prevalence of Right Ventricular Pacing-Induced Cardiomyopathy. J Cardiovasc Electrophysiol. 2016;

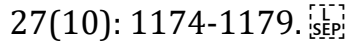

[30] Youn HJ, Park CS, Cho EJ, Junk HO, Jeon HK, Lee JM, et al. Left bundle branch block disturbs left anterior descending coronary artery flow: study using transthoracic Doppler echocardiography. J Am Soc Echocardiogr. 2005; 18(10): 1093-1098.

[31] Fang F, Chan JY, Lee AP, Sung SH, Luo XX, Jiang X, et al. Improved coronary artery blood flow following the correction of systolic dyssynchrony with cardiac resynchronization therapy. International Journal of Cardiology. 2013; 167(5): 2167-2171. 